\title{
Perspective
}

PERSPECTIVE Actualité en histoire de l'art

$1 \mid 2020$

Japon

\section{Le magazine, support originel de la photographie japonaise dans les années 1960-1970}

The Magazine, the Original Vehicle of Japanese Photography in 1960s and 1970s

Die Zeitschrift, ursprüngliches Medium der japanischen Fotografie in den

1960er-1970er Jahren

Il magazine, supporto originale della fotografia giapponese negli anni ' 60 e '70

El magazín, soporte original de la fotografía japonesa en los años 1960-1970

\section{Lilian Froger}

\section{OpenEdition}

\section{Journals}

Édition électronique

URL : http://journals.openedition.org/perspective/19172

DOl : 10.4000/perspective.19172

ISSN : 2269-7721

Éditeur

Institut national d'histoire de l'art

Édition imprimée

Date de publication : 5 juin 2020

Pagination : 281-292

ISBN : 978-2-917902-89-9

ISSN : $1777-7852$

Référence électronique

Lilian Froger, « Le magazine, support originel de la photographie japonaise dans les

années 1960-1970 », Perspective [En ligne], 1 | 2020, mis en ligne le 30 décembre 2020, consulté le 25 janvier 2021. URL : http://journals.openedition.org/perspective/19172 ; DOI : https://doi.org/10.4000/ perspective.19172 


\title{
Le magazine, support originel de la photographie japonaise dans les années 1960-1970
}

\author{
Lilian Froger
}

En mars 1974 débute à New York l'exposition New Japanese Photography, organisée au Museum of Modern Art (MoMA) par Yamagishi Shōji et par John Szarkowski, directeur du département de la photographie dans le musée. Le public américain découvre alors les clichés de quinze photographes contemporains - parmi lesquels Domon Ken, Fukase Masahisa, Kawada Kikuji, Moriyama Daidō, ou encore Tōmatsu Shōmei -, aujourd'hui considérés comme des figures majeures de la photographie japonaise de la seconde moitié du Xx $x^{e}$ siècle. D'un point de vue symbolique, le MoMA fait figure d'instance de légitimation, et après cette première exposition hors du Japon au sein de l'institution la plus respectée en matière de photographie, la production japonaise intègre rapidement le circuit des grands musées occidentaux, donnant lieu à diverses expositions collectives ${ }^{1}$ ou monographiques à la fin des années 1970, puis pendant les décennies 1980 et 1990. En Europe et aux États-Unis, les musées font simultanément l'acquisition de tirages pour leurs collections, se concentrant sur les années 1960 et 1970, admirées comme l'âge d'or de la production nippone ${ }^{3}$. La photographie japonaise existe désormais sur la scène internationale.

Avec les années 2000, l'attention se déplace sur un nouveau support : le livre de photographies. Le livre comme espace de présentation de l'image photographique éveille alors l'intérêt d'un nombre croissant de chercheurs et de commissaires d'exposition, notamment autour de la question du rapport texte/image ${ }^{4}$. Concernant plus spécifiquement la production japonaise, un premier événement important consiste en la réédition en fac-similé de plusieurs publications devenues canoniques, regroupées dans la Japanese Box ${ }^{5}$. La parution du premier volume des anthologies de Gerry Badger et de Martin Parr sur le livre de photographies, dans lequel les auteurs consacrent un chapitre entier à ces ouvrages encore mal connus hors du Japon, installe véritablement l'idée du livre comme forme caractéristique et essentielle de la production japonaise ${ }^{6}$. Les ouvrages de Gerry Badger et Martin Parr adoptent une maquette articulant une iconographie fournie et des notices concises, structure que l'on retrouve dans la plupart des autres ouvrages consacrés aux livres de photographies. Ivan Vartanian et Kaneko Ryūichi font un choix de présentation similaire dans Les Livres de photographies japonais des années 1960 et 1970, première étude en 


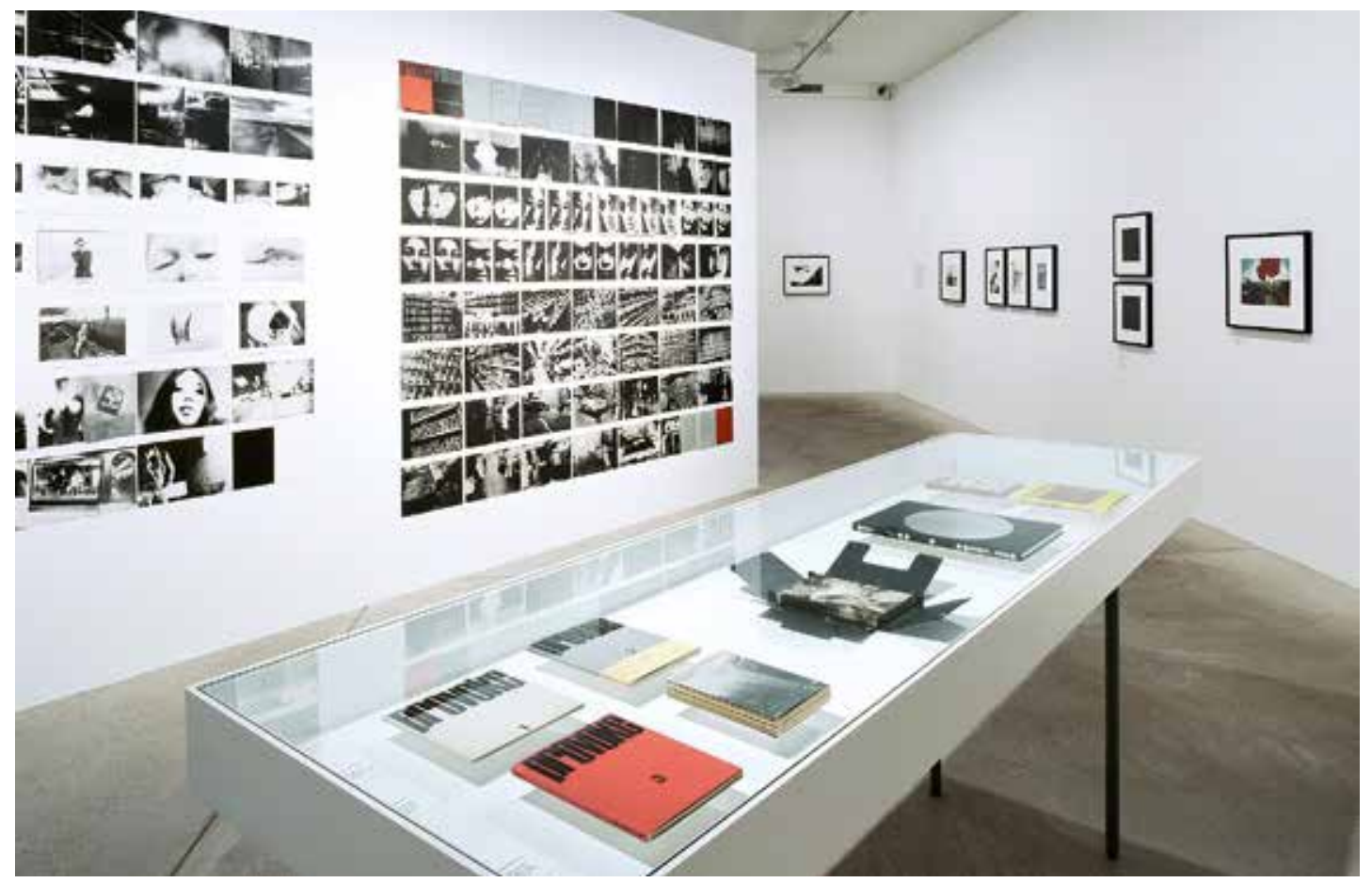

1. Vue de l'exposition Provoke. Between Protest and Performance: Photography in Japan 1960-1975, Winterthur, Fotomuseum, 2016. langue occidentale exclusivement centrée sur le sujet ${ }^{7}$, tout comme Manfred Heiting dans son livre The Japanese Photobook 1912-1990, paru récemment ${ }^{8}$. Certaines études se concentrent plutôt sur un titre en particulier, analysé en détail, à l'instar des volumes des éditions Errata sur le livre Toshi-e [Vers la ville] (1974) de Takanashi Yutaka ou bien sur Shokuji [Repas] (1993) d'Araki Nobuyoshi'. Ce travail de valorisation du livre de photographies japonais a, dans un premier temps, principalement lieu en dehors du contexte académique, du moins en Occident ${ }^{10}$ : Ivan Vartanian est éditeur, Manfred Heiting est collectionneur, Jeffrey Ladd (des éditions Errata) est d'abord photographe, Martin Parr est photographe et collectionneur de livres. Ces auteurs ont cependant en commun de défendre l'idée que la photographie japonaise est avant tout à envisager à travers le support imprimé, les photographes japonais depuis l'après-guerre ayant fait du livre la forme la plus aboutie de leur travail, au détriment de l'exposition (et par extension du tirage). Au-delà de la seule sphère japonaise, ces livres sont considérés depuis une décennie et demie comme des jalons essentiels de l'histoire mondiale de la photographie, ce que Martin Parr se plaît à répéter : « Une des plus grandes réussites de l'après-guerre est due aux photographes japonais dans les années 1960 et 1970 qui ont inventé un langage complètement nouveau et une nouvelle manière de considérer la photographie. Leurs livres étaient d'une telle qualité et d'une telle envergure qu'ils devançaient largement le reste du monde - assurément comparés à l'Amérique où l'on faisait ces livres ennuyeux avec deux pages blanches avec une photo sur la droite ${ }^{11}$."

D'abord par le biais d'expositions, puis grâce à une meilleure connaissance de l'histoire du livre de photographies, le Japon s'est imposé comme une composante majeure du monde de la photographie depuis une trentaine d'années. Le succès - critique et commercial - de certains photographes attachés à l'imprimé, comme Araki Nobuyoshi, a contribué 
à nourrir cet engouement ${ }^{12}$. Pourtant, un vaste pan de la photographie imprimée japonaise reste inexploré, et mérite qu'on s'y attarde davantage : le magazine. En effet, les revues ont souvent été le premier support d'existence des photographies, avant même leur publication sous forme de livres. Chez Araki, par exemple, la pratique photographique et la publication en périodique sont à ce point indissociables que le critique Takazawa Kenji parle dans son cas d'une "photographie de magazine ${ }^{13}$ ". La prise en compte de ces magazines, longtemps négligés, s'avère indispensable pour comprendre les spécificités de la photographie japonaise telle qu'elle s'élabore dans les années 1960 et 1970, le périodique jouant un double rôle, à la fois vecteur de diffusion de la photographie et support de création et de réflexion à part entière.

\section{Provoke, une revue à l'aura mythique}

Provoke est le titre de périodique qui revient inlassablement dès qu'il est question de photographie japonaise. Fondée en 1968 par le photographe et critique Taki Kōji, le poète et critique Okada Takahiko, ainsi que les photographes Nakahira Takuma et Takanashi Yutaka, la revue a pourtant eu une vie courte, n'ayant publié que trois livraisons avant la dissolution du groupe en 1969. Elle doit cependant sa renommée au prestige de ses créateurs, encore jeunes à l'époque mais qui deviennent des acteurs importants de la photographie japonaise à partir des années 1970, d'autant plus lorsque Moriyama Daidō rejoint le groupe pour le deuxième numéro. Pendant longtemps, l'apport de Provoke a été réduit à une esthétique, synthétisée en trois adjectifs, are, bure, boke (" brut ", "flou ", " granuleux "), trois mots presque magiques qui ont servi à résumer l'apport de Provoke à l'histoire de la photographie japonaise ${ }^{14}$, et plus globalement à définir la photographie japonaise d'après-guerre. C'était par exemple l'angle choisi par l'exposition Rough, Blurred, and Out of Focus: Provoke Magazine and Postwar Japanese Photography, présentée à l'Art Institute of Chicago de janvier à février 2012. Plus récemment, en 2019, l'exposition The Gaze of Things. Japanese Photography in the Context of Provoke a réuni le travail de quinze photographes, pas nécessairement issus du groupe Provoke, tels que Kawada Kikuji, Hosoe Eikoh, Nishimura Tamiko ou Satō Akira ${ }^{15}$. La fourchette chronologique choisie pour l'exposition (des années 1950 à 2000) allait elle aussi bien au-delà des deux années d'existence de Provoke.

Souvent citée, la revue est longtemps restée assez mal connue. Au Japon, plusieurs personnes ont pourtant œuvré dans les années 1990 à une meilleure compréhension de Provoke, sans se limiter à des questions formelles, pour au contraire prendre aussi en compte son contexte de production. On peut mentionner le travail de l'historien de la photographie

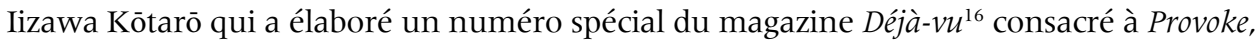
ou encore le livre Naze imada Purovōku ka? Moriyama Daidō, Nakahira Takuma, Araki Nobuyoshi no tōjō [Pourquoi encore maintenant Provoke ? L'entrée en scène de Moriyama Daidō, Nakahira Takuma et Araki Nobuyoshi] de l'éditeur et critique Nishii Kazuo ${ }^{17}$. Ces recherches n'ont cependant pas franchi les frontières de l'archipel, principalement en l'absence de traduction en anglais, et le terme " Provoke " a commencé à désigner des clichés flous en noir et blanc, fortement contrastés, sans forcément de lien avec la revue. La présence de Provoke dans plusieurs anthologies sur le livre de photographies ou sur les revues d'artistes ${ }^{18}$ au début des années 2000 a aussi contribué à sa renommée postérieure, ainsi qu'à stimuler l'intérêt à la fois des collectionneurs et des institutions.

L'exposition Provoke. Between Protest and Performance: Photography in Japan 1960-1975 à l'Albertina à Vienne en 2016, qui a circulé ensuite en Europe et à Chicago ${ }^{19}$, a cherché à complexifier la lecture de la revue Provoke, afin de ne plus la considérer seulement comme 
une coquille attrayante et fascinante. Y étaient exposés des tirages, de nombreux livres et publications, visant à présenter Provoke comme un trait d'union entre les mouvements de protestation de la fin des années 1960 et le développement de la performance, c'est-à-dire comme un périodique pleinement inscrit dans son contexte politique et artistique ${ }^{20}$. Dans l'exposition, le contenu de la revue était disposé en intégralité au mur (une fois cassée la reliure de plusieurs exemplaires), double page après double page (fig. 1). Contrairement à de nombreuses expositions - même celles spécifiquement consacrées à l'imprimé -, qui présentent les livres et revues sous vitrine, et où le visiteur doit se contenter de la couverture ou d'une seule double page, l'exposition Provoke permettait enfin de voir réellement la revue dans sa totalité. Il était alors possible d'apprécier les séquences d'images conçues par les photographes et d'évaluer comment les différentes contributions interagissaient les unes avec les autres, tout en saisissant l'importance laissée aux pages de texte en début et fin de numéro. Le catalogue de l'exposition, épais volume de près de sept cents pages, reproduit lui aussi les trois numéros dans leur ensemble, tout en les accompagnant d'un vaste appareil d'analyses, ce qui en fait un outil indispensable pour l'étude de la revue ${ }^{21}$ : traductions de textes parus dans Provoke ou écrits par ses fondateurs, traductions d'autres textes d'époque, entretiens, contributions d'historiens de la photographie, reproduction d'autres publications des années 1960 et 1970, etc. Peu de temps après, les éditions Nitesha ont imprimé en fac-similé les trois numéros de Provoke, ceux-ci étant difficilement consultables car conservés par peu de bibliothèques ${ }^{22}$.

\section{À l'ombre de Provoke}

La fin des années 1960 et la décennie 1970 sont une période d'effervescence pour la photographie au Japon, dans une sorte d'actualisation de modèles antérieurs privilégiant la forme imprimée comme support de diffusion des images et des idées ${ }^{23}$. Parallèlement à Provoke, plusieurs autres revues indépendantes sont créées, directement par des photographes, même si elles n'ont jusqu'à présent pas fait l'objet de beaucoup d'attention. Dès 1986, Kaneko Ryūichi, futur conservateur au musée de la photographie de Tōkyō, publiait avec deux autres auteurs une première étude sur les groupes de photographes indépendants : Indipendento fotogurafāzu in Japan 1976-8324. Très complète, cette publication recensait les groupes actifs à Tōkyō et hors de la capitale, les ouvertures de galeries indépendantes ainsi que les créations de revues, parmi lesquelles Shashin kōka magazine et Workshop. Elle reste à ce jour la publication la plus documentée sur les jeunes photographes de cette période.

En comparaison de l'intérêt que suscite Provoke depuis des années, les autres revues indépendantes demeurent un domaine inexploré par les expositions et par la recherche. Le catalogue de l'exposition The History of Japanese Photography - dont le titre traduit toute l'ambition - ne leur consacre par exemple que quelques pages en fin d'ouvrage, et encore sont-elles mêlées aux magazines plus grand public édités par de puissants groupes de presse (Asahi, Mainichi, Sankei) ou des fabricants de matériel photographique (Minolta fait paraître le magazine Rokkor à partir de 1955) ${ }^{25}$. L'exposition For a New World to Come. Experiments in Japanese Art and Photography, 1968-1979, organisée au Museum of Fine Arts de Houston en 2015, fait figure d'exception récente ${ }^{26}$. Elle incluait plusieurs revues (KEN, Chihei, Workshop) publiées en marge des institutions (musées ou grands éditeurs), que Nakamori Yasufumi a regroupées sous l'expression de "voix décentrées ${ }^{27}$ ". Encore trop peu étudiés, ces périodiques ont été des supports de publication de photographies, mais il ne faut pas perdre de vue qu'ils ont aussi été les principaux vecteurs de diffusion des débats de l'époque, qu'ils soient d'ordres politique ou plus artistique, à l'image 
du premier numéro de la revue $K E N$ qui est une critique acerbe de l'Expo'70 d'Ōsaka et de son utilisation par le gouvernement pour faire oublier le mécontentement étudiant, syndical et social. Les nombreux textes critiques qu'ils contiennent évoquent ainsi la reproductibilité et la stabilité relative de l'image photographique, mettent en cause le rôle du photographe en tant que producteur d'images dans la société médiatique des années 1970, de même qu'ils reconsidèrent les notions de réel et de narration ${ }^{28}$.

\section{Les magazines grand public, indispensables relais}

Les revues indépendantes ont fait l'objet de peu d'études, en raison de leur faible diffusion à l'époque de leur parution, qui fait qu'elles sont relativement peu représentées dans les collections des musées et des bibliothèques ${ }^{29}$. Paradoxalement, les magazines grand public, malgré des tirages de plusieurs dizaines voire centaines de milliers d'exemplaires, sont tout aussi peu présents dans

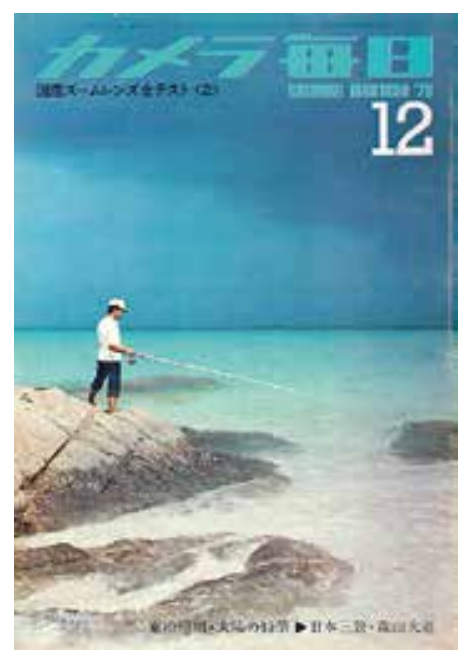

2. Première de couverture de la revue Kamera mainichi, $\mathrm{n}^{\circ} 242$, décembre 1973, avec Tōmatsu Shōmei, série « Taiyō no enpitsu » [Le crayon du soleil]. les collections publiques ${ }^{30}$. Leur durée de vie est souvent courte, limitée à quelques mois, sans être conservés, à l'inverse des livres. Livres de photographies et magazines grand public (spécialisés en photographie ou bien plus généralistes) sont pourtant intimement liés. Pendant les années 1960, se met ainsi en place un système de publication de séries photographiques, d'abord prépubliées dans la presse en épisodes de deux à seize pages environ, avant de bénéficier d'une parution en volume sous forme de livre. Yamagishi Shōji, influent rédacteur en chef de la revue Kamera mainichi, va tout particulièrement promouvoir ce fonctionnement ${ }^{31}$, adapté à partir de celui des revues littéraires. Le magazine devient alors le relais incontournable entre le photographe, ses clichés, les lecteurs et le futur livre à venir. Le contenu du livre Taiyō no enpitsu de Tōmatsu Shōmei avait ainsi d'abord existé sous la forme de plusieurs portfolios dans les pages de Kamera mainichi avant que les photographies ne soient réunies en un volume en $1975^{32}$ (fig. 2).

Grand connaisseur des magazines de photographie japonais - qu'il a lui-même collectés depuis les années 1960 -, Kaneko Ryūichi est catégorique :

Vous pouvez regarder partout dans le monde : il n'y a rien de véritablement comparable à ces revues japonaises de l'époque. Ce qui était unique dans ces revues [commerciales], c'était l'idée d'une série sur un thème. Elles exploraient un thème spécifique sur le cours d'une année au gré de chaque numéro. Si vous publiiez un portfolio dans l'une de ces revues, vous aviez près de 120 pages d'images publiées, à raison d'une dizaine par numéro, ce qui équivaut, en fin de compte, à un livre de photographie. Ce fut le cas des années 1950 jusqu'au début des années 1980. Donc dans un sens, les magazines de photographie étaient devenus une base à partir de laquelle on pouvait faire un livre. Pour un photographe, retrouver son travail dans l'un de ces magazines était une étape importante. Publier un livre de photographie était alors considéré comme l'étape suivante, et c'est la culture des magazines qui a nourri celle des livres de photographie ${ }^{33}$.

Alors que si peu d'ouvrages ont vu le jour sur la question spécifique du magazine ${ }^{34}$, la parution prévue pour le printemps 2020 de The History of Japanese Photo Magazines, 1880s to 1980 s devrait en partie relancer l'intérêt pour la question ${ }^{35}$. L'étude des publications en magazine permet en effet de renouveler la connaissance de la photographie japonaise, 
même concernant certains grands noms sur lesquels on pensait tout savoir. Les éditions Getsuyōsha ont ainsi réuni l'intégralité des portfolios publiés par Moriyama Daidō ${ }^{36}$ et Nakahira Takuma ${ }^{37}$ depuis le tout début de leur carrière et jusqu'à la fin des années 1970. Ces anthologies reproduisent à l'échelle 1 l'ensemble de ces pages, extraites d'Asahi kamera, Kamera mainichi, Asahi jānaru et d'autres périodiques plus confidentiels. Au fil des pages, on retrouve certaines images devenues iconiques et on remarque clairement comment ces séries fragmentées se poursuivent sur plusieurs numéros, voire parfois dans plusieurs titres de magazines différents, pour être ensuite à nouveau mélangées et reprises dans leurs livres.

\section{Enrichir les corpus connus}

Plonger dans les publications en magazine - comme le permettent ces anthologies -, c'est aussi ouvrir la voie à de nouveaux corpus d'images moins balisés. On y découvre chez Moriyama et Nakahira de nombreuses images en couleur datant des années 1960 et 1970. L'usage de la couleur permet à Nakahira de renouveler sa vision de la ville et renforce le caractère mystérieux de ce qu'il découvre avec son appareil, remplaçant le flou et le bougé par des couleurs passées, comme délavées, associées à des zones sombres qui rendent les clichés parfois difficilement lisibles (fig. 3a-b). Ces images vont à rebours de l'histoire de la photographie japonaise d'après-guerre telle qu'elle a été écrite jusqu'à présent, c'est-à-dire pensée comme une histoire du noir et blanc. Cette remarque vaut aussi pour l'anthologie des publications périodiques de Walker Evans, qui réunissait entre autres les portfolios publiés par le photographe dans le magazine Fortune, intégralement en couleur, alors qu'on associe plus volontiers le travail d'Evans à la photographie monochrome ${ }^{38}$.

Le même constat est valable pour Fukase Masahisa. Photographe majeur des années 1960 et 1970, il vient de faire l'objet d'une volumineuse publication conçue par Kosuga Tomo, qui gère les archives du photographe depuis son décès ${ }^{39}$. L'ouvrage s'appuie sur les innombrables parutions du travail de Fukase dans des magazines pour mieux apprécier l'évolution de sa pratique photographique, de ses tout premiers portfolios jusqu'à la célèbre série Karasu [Les corbeaux], publiée par la suite en 1986 sous forme de livre ${ }^{40}$. Tous les clichés de cette série sont cependant parus précédemment dans la revue Kamera mainichi, à la demande de Yamagishi Shōji, entre octobre 1976 et novembre 1982. Ces portfolios constituent ainsi la première présentation des clichés de cette série en train de se faire et révèlent certaines images méconnues, écartées par la suite lors de la mise en page du livre.

Comme pour beaucoup de ses contemporains, le magazine constitue pour Fukase une première étape indispensable pour la diffusion de ses clichés autant que pour l'expérimentation. À peine ses photographies sont-elles prises qu'elles sont imprimées dans les pages de divers magazines. Au moment de penser la publication d'un livre (plus rarement sous forme d'exposition), il recompose des séquences d'images, mélange d'anciens clichés et complexifie progressivement la narration. Ce travail d'édition chez les photographes est d'ailleurs de plus en plus considéré dans les ouvrages et catalogues sur la photographie japonaise, à mesure que ces corpus imprimés en magazines sont explorés ${ }^{41}$. Comme c'était le cas avec Moriyama ou avec Nakahira, en parcourant l'ouvrage consacré aux portfolios en magazine de Fukase, on découvre une surprise de taille : de nombreux clichés

3a-b. Nakahira Takuma, deux doubles pages de la série « Hakubutsu-zukan. Toshi » [Un dictionnaire illustré d'histoire naturelle. La ville], publiées dans Kamera mainichi, no 226, août 1972. 

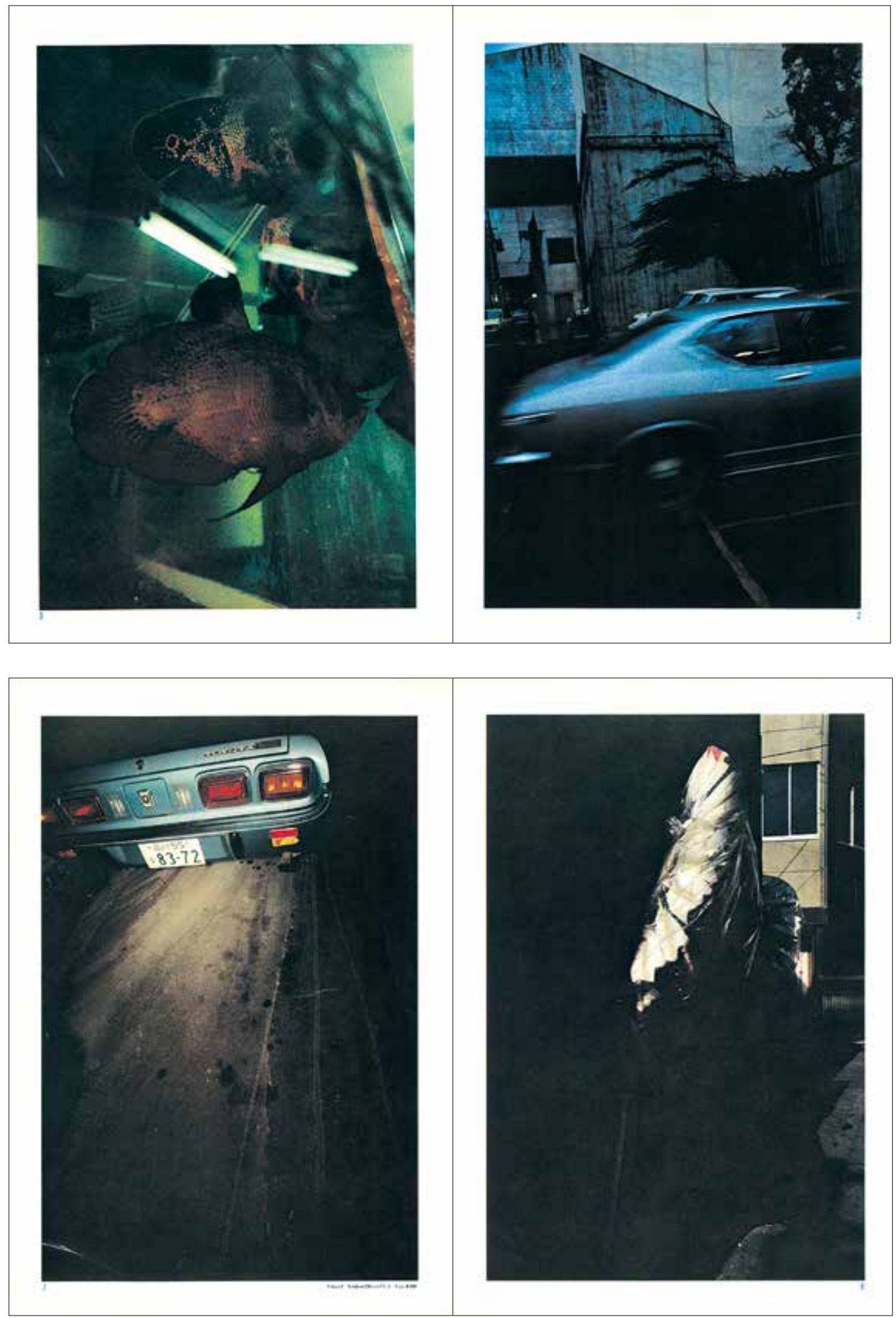


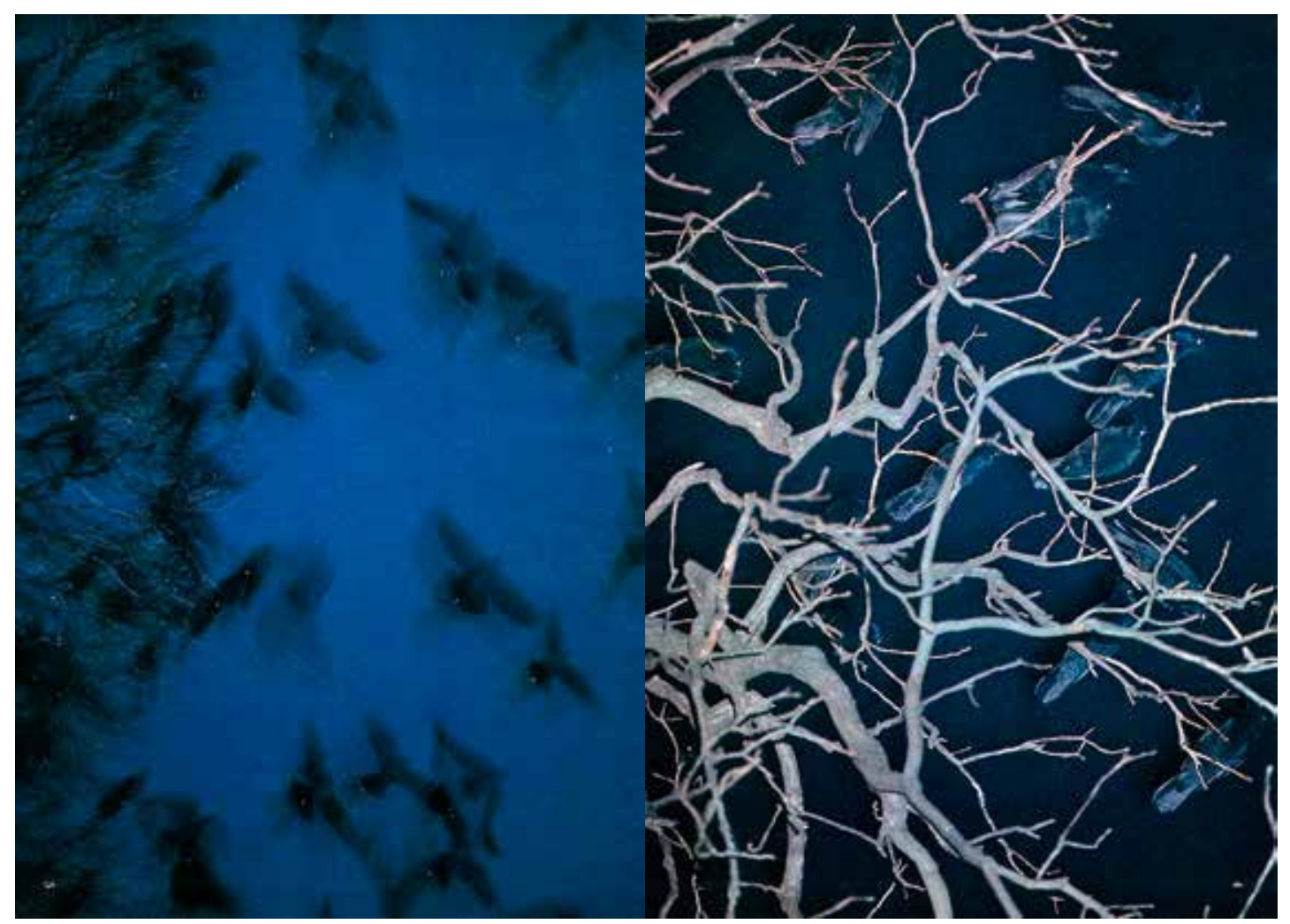

4. Fukase Masahisa, double page de la série "Karasu. 4 » [Les corbeaux. 4], publiée dans Kamera mainichi, $\mathrm{n}^{\circ} 297$, juin 1978 en couleur, même au sein de la série Karasu, alors que le livre de 1986 était paru exclusivement en noir et blanc, tout comme les tirages qui ont ensuite intégré les collections des musées (fig. 4). En raison de l'insatisfaction de Fukase vis-à-vis du rendu des images polychromes au développement, celui-ci n'a jamais fait de tirages de ses images imprimées en couleur en magazine ${ }^{42}$. Et de fait, les tirages en couleur du photographe sont quasi inexistants, ce qui a conduit à penser que la production de Fukase était seulement monochrome.

Depuis une quinzaine d'années, l'intégration du livre de photographies a complété la connaissance de l'histoire de la photographie japonaise, mais il reste encore à l'élargir pleinement au magazine, acteur principal de la chaîne éditoriale et de diffusion des clichés. Moriyama affirme sans hésiter que dans les années 1960, "les opportunités de montrer [son] travail étaient à quasiment $100 \%$ restreintes aux magazines ${ }^{43}$. » Ignorer les magazines conduit sans aucun doute à se couper d'une partie de la production de ces photographes. En effet, dans les années 1960 et 1970, les photographes japonais se désintéressent de la question du tirage, celui-ci n'étant souvent considéré que comme un outil de travail destiné à préparer une future mise en page. Les photographies finalement tirées étant ainsi moins nombreuses que celles publiées, il semble donc hasardeux de se focaliser sur les tirages comme autant de " chefs-d'œuvre ". La problématique de la couleur est également révélatrice. En raison de coûts encore élevés et du manque de stabilité des procédés de développement couleur dans les années 1960 et 1970, on ne conserve que très peu de tirages polychromes des photographes japonais de cette époque, 
ce qui a longtemps conduit à penser qu'ils ne travaillaient qu'en noir et blanc. À l'inverse, l'impression en couleur était techniquement mieux maîtrisée et les grands groupes de presse pouvaient surtout dépenser des sommes importantes dans l'impression de pages en couleur très appréciées de leurs lecteurs. Pour ces raisons, la couleur est présente très tôt dans la production de Moriyama, Nakahira, Tōmatsu, Fukase ou Ueda - pour ne citer que quelques grands noms -, et c'est dans les magazines que ces images en couleur ont pu exister. Ainsi, s'intéresser au magazine permet d'intégrer aux récits établis des images aussi nombreuses qu'inédites, bien au-delà de ce qui a jusqu'à présent été retenu par les musées pour la constitution de leurs collections ${ }^{44}$. 


\section{Lilian Froger}

Lilian Froger est docteur en histoire de I'art contemporain, auteur d'une thèse sur le livre de photographies japonais. Il a bénéficié en 2017 du soutien du CNAP pour un projet de recherche sur l'exposition de la photographie au Japon dans les années 1970. Il est actuellement chargé des collections de photographies contemporaines au musée d'Art et d'Archéologie d'Aurillac.

\section{NOTES}

1. Par exemple: Neue Fotografie aus Japan à la Kulturhaus de Graz en 1977 ; Japan: A Self-Portrait à I'International Center of Photography (New York) en 1979 ; Black Sun: The Eyes of Four. Roots and Innovation in Japanese Photography à la Serpentine Gallery (Londres) en 1986. Alain Sayag inclut par ailleurs une section photographique à la grande exposition 1910-1970 : Japon des avantgardes au Centre Pompidou en 1986, dans laquelle on retrouve une grande partie des photographes déjà présentés au MoMA quelques années auparavant.

2. Shomei Tomatsu: Japan 1952-1981 au Forum Stadtpark de Graz en 1984 ; Nobuyoshi Araki, Journal Intime à la Fondation Cartier pour l'art contemporain en 1984 ; Daido Moriyama: Stray Dog au Museum of Modern Art de San Francisco en 1999.

3. À l'exception de quelques tirages de Hamaya Hiroshi, Kuwabara Kineo, Suzuki Hachirō et Ueda Shōji, le Centre Pompidou ne conserve par exemple aucune photographie antérieure aux années 1960. La période des années 1960-1970 est surreprésentée dans la plupart des collections occidentales et, en comparaison, il est plus difficile de trouver des clichés japonais pictorialistes ou bien modernistes hors des collections japonaises, publiques et privées.

4. Voir Philippe Arbaïzar, "Le livre de photographe », dans Les Cahiers du Musée national d'art moderne, no 81, automne 2002, p. 36-61; François Brunet, "Photography and the Book », dans Photography and Literature, Londres, Reaktion Books (coll. «Exposures »), 2009, p. 34-62 ; Michelle Debat (dir.), La Photographie et le livre, Paris, Trans Photographic Press, 2003 ; Danièle Méaux (dir.), Livres de photographies et de mots, Caen, Lettres modernes Minard, 2009 ; Andrew Roth (dir.), The Book of 101 Books. Seminal Photographic Books of the Twentieth Century, New York, PPP Editions, 2001 ; Andrew Roth (dir.), The Open Book. A History of the Photographic Book from 1878 to the Present, Göteborg, Hasselblad Center, 2004. En France, les études d'Anne Moeglin-Delcroix sur le livre d'artiste servent de modèle épistémologique à ces premières recherches.

5. Christoph Schifferli (dir.), The Japanese Box, Göttingen / Paris, Steidl / 7L, 2001. Ce coffret réunit les livres Kitarubeki kotoba no tame ni [Pour un nouveau langage à venir] (1970) de Nakahira Takuma, Senchimentaruna tabi [Voyage sentimental] (1971) d'Araki Nobuyoshi, Shashin yo, sayōnara [Au revoir à la photographie]
(1972) de Moriyama Daidō, ainsi que les trois numéros de la revue Provoke.

6. Gerry Badger, Martin Parr, « De la provocation à la réflexion : le livre de photographies japonais depuis la Seconde Guerre mondiale ", chap. IX, dans Le Livre de photographies. Une histoire, Virginie de Bermond-Gettle, Anne-Marie Terrel (trad. fra.), vol. 1, Paris, Phaidon, 2005, p. 266-311.

7. Kaneko Ryūichi, Ivan Vartanian, Les Livres de photographies japonais des années 1960 et 1970, Pierre Saint-Jean (trad. fra.), Paris, Seuil, 2009.

8. Manfred Heiting (dir.), The lapanese Photobook 19121990, Göttingen, Steidl, 2017.

9. Jeffrey Ladd (dir.), Toshi-e (Towards the City). Yutaka Takanashi, New York, Errata editions, 2010 ; Jeffrey Ladd (dir.), The Banquet. Nobuyoshi Araki, New York, Errata editions, 2012.

10. Au Japon, les historiens lizawa Kōtarō et Kaneko Ryūichi avaient déjà écrit dans les années 1990 sur le livre de photographies - japonais comme occidental mais leurs ouvrages et articles n'ont eu à l'époque qu'un retentissement limité. C'est véritablement l'ouvrage que Kaneko publie avec Ivan Vartanian en 2009 qui contribue à mettre en avant ses recherches auprès d'un plus large public, porté par l'intérêt général pour le livre de photographies. Concernant leurs écrits antérieurs, voir par exemple : lizawa Kōtarō (dir.), Shashinshū wo yomu [Lire les livres de photographies], vol. 1, Tōkyō, Metarōgu, 1997 ; lizawa Kōtarō, Shashinshū no tanoshimi [Le plaisir des livres de photographies], Tōkyō, Asahi shinbunsha, 1998 ; lizawa Kōtarō (dir.), Shashinshū wo yomu [Lire les livres de photographies], vol. 2, Tōkyō, Metarōgu, 2000 ; Kaneko Ryūichi, " "Shashinshū" no keitai ni tsuite " [Le livre de photographies comme forme], dans FROG, vol. 3, no 9, septembre 1991, p. 3.

11. Rémi Coignet, entretien avec Martin Parr, dans Conversations 2, Paris, The Eyes Publishing, 2016, p. 170.

12. Sur les livres de photographies d'Araki, voir : lizawa Kōtarō, Araki-bon! 1970-2005 [Les livres de photographies d'Araki. 1970-2005], Tōkyō, Bijustu shuppansha, 2006 ; Kohara Masashi, Nagahara Kōji (dir.), Shashinshūkyō Arākī [Araki, le fou de livres de photographies], cat. exp. (Nagaizumi-chō, Izu Photo Museum, 2012), Nagaizumi-chō, Izu Photo Museum / Nohara, 2012.

13. Takazawa Kenji, "Araki Goroku. Shigan to higan » [Araki Goroku. Le monde terrestre et le paradis], entretien avec Araki Nobuyoshi, dans Photographica, vol. 21, printemps 2011, p. 168 : "Araki-san no shashin wa zasshi-teki ».

14. Selon Shimizu Minoru, "l'étiquette "granuleux, brut, flou" collée au travail de Moriyama a cependant été associée au magazine Provoke ». Shimizu Minoru, " 'Grainy, Blurry, Out-of-Focus': Daido Moriyama's Farewell Photography ", dans Simon Baker (dir.), Daido Moriyama, cat. exp. (Londres, Tate Modern 2012-2013), Londres, Tate Publishing, 2012, p. 57 : «However, the label attached to Moriyama's work "grainy, blurry, outof-focus" became attached to the magazine Provoke ».

15. Les textes de Miryam Sas ("Conceptualizing Japanese Post-War Photography: Snap, Movement, Refusal », p. 201-206) et de Yasumi Akihito (« Resisting Aesthetics: 
Body, Image, Matter », p. 215-221) dans le catalogue contextualisaient en revanche précisément la période des années 1960-1970. Nuria Enguita, Myriam Sas, Akihito Yasumi, The Gaze of Things. Japanese Photography in the Context of Provoke, cat. exp. (Valence, Bombas Gens Centre d'Art, 2019-2020), Madrid / Valence, La Fábrica / Bombas Gens Centre d'Art, 2019.

16. Déjà-vu, no 14 : «Purovōku no jidai. Sengo shashin no tenkanten » [L'époque de Provoke, moment pivot de la photographie d'après-guerre], octobre 1994.

17. Nishii Kazuo, Naze imada Purovōku ka ? Moriyama Daidō, Nakahira Takuma, Araki Nobuyoshi no tōjō [Pourquoi encore maintenant Provoke? L'entrée en scène de Moriyama Daidō, Nakahira Takuma et Araki Nobuyoshi], Tōkyō, Seikyūsha, 1996.

18. La revue Provoke figure ainsi dans I'anthologie In Numbers. Philip E. Aarons, Victor Brand, Andrew Roth (dir.), In Numbers. Serial Publications by Artists Since 1955, Zurich, PPP éditions, 2009.

19. Exposition Provoke. Between Protest and Performance: Photography in Japan 1960-1975, Vienne, Albertina, 29 janvier - 8 mai 2016 ; Winterthur, Fotomuseum, 28 mai - 28 août 2016 ; Paris, Le Bal, 14 septembre 11 décembre 2016 ; Chicago, Art Institute of Chicago, 28 janvier - 30 avril 2017.

20. La revue Provoke était aussi dans la sélection de I'exposition Tokyo. 1955-1970: A New Avant-Garde au MoMA en 2012-2013, en tant que production importante de la scène japonaise de l'époque, dans une vision élargie qui dépassait le strict cadre photographique. En 2011, Miryam Sas, l'une des auteurs du catalogue de l'exposition new-yorkaise, avait déjà consacré un chapitre de l'un de ses livres à Provoke au sein d'une étude étendue sur les avant-gardes japonaises après 1945 : Miryam Sas, «Provoke Era: New Languages of Japanese Photography ", dans Experimentals Arts in Postwar Japan: Moments of Encounter, Engagement, and Imagined Return, Cambridge / Londres, Harvard University Asia Center, 2011, p. 180-201.

21. Diane Dufour, Duncan Forbes, Walter Moser, Matthew S. Witkovsky (dir.), Provoke. Between Protest and Performance. Photography in Japan 1960-1975, cat. exp. (Vienne, Albertina ; Winterthur, Fotomuseum ; Paris, Le Bal ; Chicago, Art Institute of Chicago, 20162017), Göttingen, Steidl, 2016.

22. Purovōku, fukkokuban [Provoke, édition fac-similé], Tōkyō, Nitesha, 2018.

23. C'est le cas notamment au moment du pictorialisme puis du modernisme, avec une revue telle que Kōga, fondée par Nojima Yasuzō et inspirée de ce que font László Moholy-Nagy, El Lissitzky ou Herbert Bayer en Europe au même moment. La montée du militarisme, la période de la guerre, puis le direct après-guerre ont aussi été propices à l'édition photographique de magazines, notamment de propagande. Sur les années 19201930, voir : Tōkyō-to shashin bijutsukan (dir.), Kōga to shinkō shashin: Modanizumu no Nihon [Kōga et la Nouvelle Photographie : le modernisme japonais], cat. exp. (Tōkyō, Tōkyō-to shashin bijutsukan, 2018), Tōkyō, Kokusho Kankōkai, 2018 ; Jelena Stojković, «Images With and Without Texts: The Photographic Magazine in 1930s Japan ", dans The Japan Society Proceedings, n 155, 2018, p. 124-133. Sur les périodiques de propagande, voir : Shirayama Mari, Kohara Masashi (dir.), Sensō to heiwa. "Hōdō-shashin " ga tsutaetakatta Nihon [Guerre et paix. Le Japon tel que raconté par le photojournalisme], cat. exp. (Nagaizumi-chō, Izu Photo Museum, 2015-2016), Tōkyō, Heibonsha, 2015.

24. Kaneko Ryūichi, Nagai Hiroshi, Shimao Shinzō, Indipendento fotogurafāzu in Japan 1976-83 [Photographes indépendants au Japon, 1976-1983], Tōkyō, Tōkyō Shoseki kabushigaisha, 1989.

25. Shirayama Mari, "Major Photography Magazines », dans Anne Wilkes Tucker, lizawa Kōtarō, John Junkerman (dir.), The History of Japanese Photography, cat. exp. (Houston, Museum of Fine Arts ; The Cleveland Museum of Art, 2003), New Haven, Yale University Press, 2003, p. 378-385.

26. Après avoir été présentée à Houston du 7 mars au 12 juillet 2015, l'exposition a circulé à la Grey Art Gallery, New York University (11 septembre - 5 décembre 2015) et à la Japan Society Gallery à New York (9 octobre 11 janvier 2016).

27. Nakamori Yasufumi, "Decentered Voices. PostProvoke Independent Photography Magazines ", dans Nakamori Yasufumi, Allison Pappas (dir.), For a New World to Come. Experiments in Japanese Art and Photography, 1968-1979, cat. exp. (Houston, Museum of Fine Arts ; New York, New York University, Grey Art Gallery; The Japan Society Gallery, 2015-2016), Houston, Museum of Fine Arts, 2015, p. 50-59.

28. Les écrits critiques de Nakahira Takuma ont ainsi d'abord connu une première publication dans des magazines à grand tirage ou plus indépendants avant d'être rassemblés dans divers recueils. Pour des exemples de traductions d'écrits de photographes de la période, voir : Ivan Vartanian (dir.), Setting Sun. Writings by Japanese Photographers, New York, Aperture, 2006 ; Chong Do-Ryun, Hayashi Michio, Kajiya Kenji, Sumimoto Fumihiko (dir.), From Postwar to Postmodern. Art in Japan 1945-1989. Primary Documents, New York, MoMA, 2012.

29. Le musée de la photographie de Tōkyō fait figure d'exception. Le fait que Kaneko Ryūichi, historien de la photographie et grand collectionneur de livres et de magazines, ait intégré l'équipe du musée dès la période de préfiguration a sans aucun doute joué un rôle important dans la constitution de la collection du musée. Dans un guide des musées consacrés à la photographie - publics et privés - paru en 2002, il est précisé que le musée de la photographie de Tōkyō conserve 18000 tirages et 47650 imprimés, dont 23175 magazines. Matsumoto Norihiko, Nihon no bijutsukan to shashin korekushon [Musées et collections de photographies au Japon], Tōkyō, Tōkyō-to shashin bijutsukan / Tankōsha, 2002. Voir aussi : Ivan Vartanian, "Collecting the Japanese Photobook », entretien avec Kaneko Ryūichi, dans The Photobook Review, no 8 , printemps 2015, p. 10. Aux États-Unis, le Museum of Fine Arts de Houston possède une collection conséquente d'imprimés, en raison de l'acquisition d'une partie de la bibliothèque de Manfred Heiting, constituée de nombreux périodiques japonais.

30. Les bibliothèques universitaires, par le biais des abonnements souscrits pour les clubs étudiants de 
photographie, ont des collections de magazines relativement complètes à partir des années 1960. Nombreux sont aussi les musées à avoir enrichi a posteriori leurs collections de magazines pour alimenter leur fonds documentaire, les périodiques des années 1960-1970 étant encore relativement accessibles sur le marché de l'occasion.

31. Il se fait remarquer après la publication d'un supplément de cinquante-six pages au numéro d'avril 1965, intitulé Shita dashi tenshi [L'ange qui tire la langue], qui rassemble des clichés de Tatsuki Yoshihiro et des poèmes de Terayama Shūji. Par la suite, il fait le choix de confier plusieurs pages aux photographes plutôt que de publier des images individuelles.

32. Tōmatsu Shōmei, Taiyō no enpitsu. Okinawa, umi to sora to shima to hitobito, soshite Tōnan Ajia e [Le crayon du soleil. Okinawa, la mer et ciel, les îles et leurs habitants, puis direction l'Asie du Sud-Est], Tōkyō, Mainichi shinbunsha, 1975.

33. Marc Feustel, "Conversation avec Ryūichi Kaneko », dans The Eyes, n 7, automne-hiver 2016, p. 126-129.

34. Le magazine de photographie japonais est rarement présent dans les études plus générales sur les périodiques artistiques. Ainsi, parmi les textes que le spécialiste Gwen Allen a réunis pour son anthologie The Magazine, seul figure un extrait du manifeste publié dans le premier numéro de Provoke. Gwen Allen, The Magazine, Cambridge / Londres, The MIT Press / Whitechapel Gallery, 2016, p. 193-194.

35. Kaneko Ryūichi, Toda Masako, Ivan Vartanian, The History of Japanese Photo Magazines, 1880s to 1980s, Tōkyō, Goliga, à paraître en 2020 en même temps qu'une version japonaise par les éditions Heibonsha. Ivan Vartanian avait écrit un premier article, annonciateur de cet ouvrage à venir : Ivan Vartanian, « Magazine Work », dans Aperture, n 219, Tokyo, été 2015, p. 28-35.

36. Moriyama Daidō, Nippon gekijō - Magazine Work 1965-1970 [Théâtre japonais - Magazine Work 19651970], Tōkyō, Getsuyōsha, 2009 ; Moriyama Daidō, Nanika e no tabi - Magazine Work 1971-1974 [Voyage vers quelque chose - Magazine Work 1971-1974], Tōkyō, Getsuyōsha, 2009.

37. Nakahira Takuma, Toshi, fūkei, zukan - Magazine Work 1964-1982 [Ville, paysage, livre illustré-Magazine Work 1964-1982], Tōkyō, Getsuyōsha, 2011. Depuis plusieurs années, Franz Prichard œuvre à mettre en lumière des pans largement méconnus du travail de Nakahira. Voir par exemple : Franz Prichard, "At the Limits of the Gaze ", dans Aperture, n 219, été 2015, p. 44-48.

38. David Campany, Walker Evans. The Magazine Work, Göttingen, Steidl, 2014. En 1960, Robert Doisneau photographie aussi en couleurs, à Palm Springs, pour le magazine Fortune. Un prochain colloque consacré à la photographie couleur abordera la production polychrome de plusieurs photographes plus volontiers associés au noir et blanc: Willy Ronis, André Kertész, Robert Capa. Colloque «Que fait la couleur à la photographie? Techniques, usages, controverses ", organisé par Nathalie Boulouch et Gilles Désiré dit Gosset, Centre culturel international de Cerisy, 19-23 août 2020.

39. Kosuga Tomo, Masahisa Fukase, Paris, Xavier Barral, 2018.
40. Fukase Masahisa, Karasu [Les corbeaux], Tōkyō, Sōkyūsha, 1986. Pour une lecture critique de cette série, voir : Philip Charrier, " "Becoming a Raven": SelfRepresentation, Narration, and Metaphor in Fukase Masahisa's "Karasu" Photographs ", dans Japanese Studies, vol. 29, no 2, 2009, p. 209-234.

41. Sur cet aspect du travail chez Tōmatsu Shōmei, voir : Kaneko Ryūichi, "Shomei Tomatsu, Organizer of Photographs ", dans Juan Vicente Aliaga (dir.), Shomei Tomatsu, cat. exp. (Barcelone, Casa Garriga Nogués, 2018), Barcelone, RM / Fundación Mapfre, 2018, p. 22-29.

42. Kosuga Tomo, "Raven Scenes ", dans Kosuga, 2018, cité n. 39, p. 272.

43. Moriyama Daidō, "Unexpected Encounters 1960s ", dans Moriyama Daidō, Moriyama Daidō: Nippon gekijō shashinchō [Moriyama Daidō : Théâtre japonais], Tōkyō, Getsuyōsha / Bookshop M, 2018, n. p. : "At that time, opportunities to show my work were confined almost 100 percent to magazines. »

44. Quand John Szarkowski organise l'exposition New Japanese Photography en 1974, presque aucun photographe n'a alors de tirages disponibles. Szarkowski choisit donc à partir de portfolios de magazines les images qu'il souhaite voir tirer pour l'exposition. Les photographes font si peu de cas de ces tirages qu'ils ne cherchent pas à les récupérer à la fin de l'exposition et les donnent au musée. Ces quelques deux cents tirages forment encore actuellement l'essentiel de la collection de photographie japonaise du MoMA. 